\title{
Single Cesium Lead Halide Perovskite Nanocrystals at Low Temperature: Fast Single- Photon Emission, Reduced Blinking, and Exciton Fine Structure
}

Gabriele Rainò, ${ }^{\dagger \dagger}$ Georgian Nedelcu, ${ }^{\ddagger}, \S$ Loredana Protesescu, ${ }^{\ddagger}, \S$ Maryna I. Bodnarchuk, ${ }^{\ddagger}, \S$ Maksym V. Kovalenko, ${ }^{*},{ }^{\ddagger}$ Rainer F. Mahrt, ${ }^{\dagger}$ and Thilo Stöferle* ${ }^{\dagger}$

${ }^{\dagger}$ IBM Research-Zurich, Säumerstrasse 4, 8803 Rüschlikon, Switzerland

${ }^{\ddagger}$ Institute of Inorganic Chemistry, Department of Chemistry and Applied Biosciences, ETH Zürich, Vladimir Prelog Weg 1, 8093 Zürich, Switzerland

${ }^{\S}$ Laboratory for Thin Films and Photovoltaics, EMPA-Swiss Federal Laboratories for Materials Science and Technology, Überlandstrasse 129, 8600 Dübendorf, Switzerland

\section{Supporting Information}

ABSTRACT: Metal-halide semiconductors with perovskite crystal structure are attractive due to their facile solution processability, and have recently been harnessed very successfully for high-efficiency photovoltaics and bright light sources. Here, we show that at low temperature single colloidal cesium lead halide $\left(\mathrm{CsPbX}_{3}\right.$, where $\left.\mathrm{X}=\mathrm{Cl} / \mathrm{Br}\right)$ nanocrystals exhibit stable, narrow-band emission with suppressed blinking and small spectral diffusion. Photon antibunching demonstrates unambiguously nonclassical single-photon emission with radiative decay on the order of $250 \mathrm{ps}$, representing a significant acceleration compared

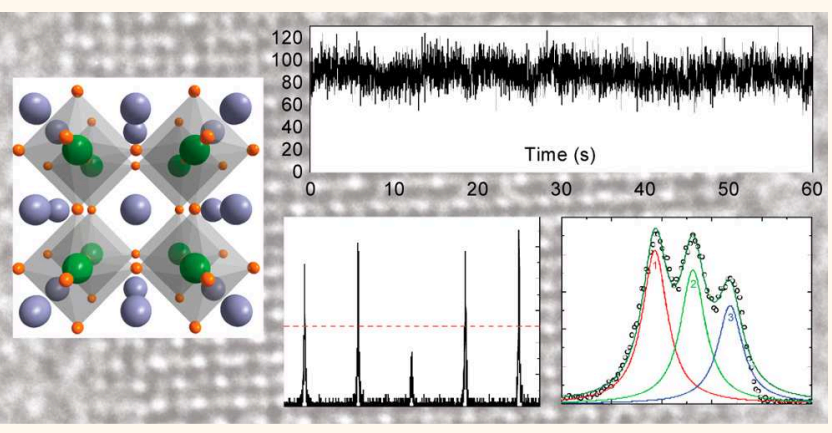
to other common quantum emitters. High-resolution spectroscopy provides insight into the complex nature of the emission process such as the fine structure and charged exciton dynamics.

KEYWORDS: perovskites, halides, quantum dots, nanocrystals, single photon sources, optoelectronics

$\mathrm{T}$ he outstanding optoelectronic properties of metalhalide perovskites, in particular hybrid organicinorganic lead halides $\left(\mathrm{MAPbX}_{3}\right.$, where $\mathrm{MA}=$ methylammonium and $\mathrm{X}=\mathrm{Br}, \mathrm{I})$ have recently fueled enormous interest in this class of materials. ${ }^{1,2}$ Inexpensive solar cells with conversion efficiencies of about $20 \%$ have been demonstrated using hybrid organic-inorganic perovskites. ${ }^{3-5}$ Astonishingly, the same material has also excellent emission properties, ${ }^{6}$ as was evidenced by optically pumped amplified spontaneous emission ${ }^{7}$ and lasing. ${ }^{8,9}$ All these applications are based on thin films that comprise microcrystalline perovskite structures. However, such ensembles of crystallites do not show strong quantum correlations between photons, even not on the level of a single microcrystal. ${ }^{10}$ Nonclassical light emission, which has been found in single organic dye molecules, ${ }^{11}$ semiconductor quantum dots ${ }^{12-14}$ and color centers in diamond ${ }^{15,16}$ and other materials, has enabled superlocalization microscopy ${ }^{17}$ and deterministic single photon sources for secure quantum communication. ${ }^{18}$ It would be tantalizing to harness the high oscillator strength, high quantum yield, efficient charge transport, solution-processability and the simplicity of synthesis of these perovskites for such applications. Furthermore, being able to access single emitters circumvents averaging effects within the ensemble of microcrystallites that can cloak fundamental light absorption and emission processes, which are of key interest in the improvement of the photovoltaic and laser devices.

Very recently, fully inorganic nanocrystals (NCs) of perovskite-type cesium lead halides $\left(\mathrm{CsPbX}_{3}, \mathrm{X}=\mathrm{Cl}, \mathrm{Br}, \mathrm{I}\right)$ have been synthesized with precise size- and compositional control, offering very bright, tunable, narrowband luminescence over the whole visible wavelength range. ${ }^{19}$ High defect tolerance and absence of detrimental mid-gap surface defects,

Received: November 20, 2015

Accepted: January 15, 2016

Published: January 15, 2016 

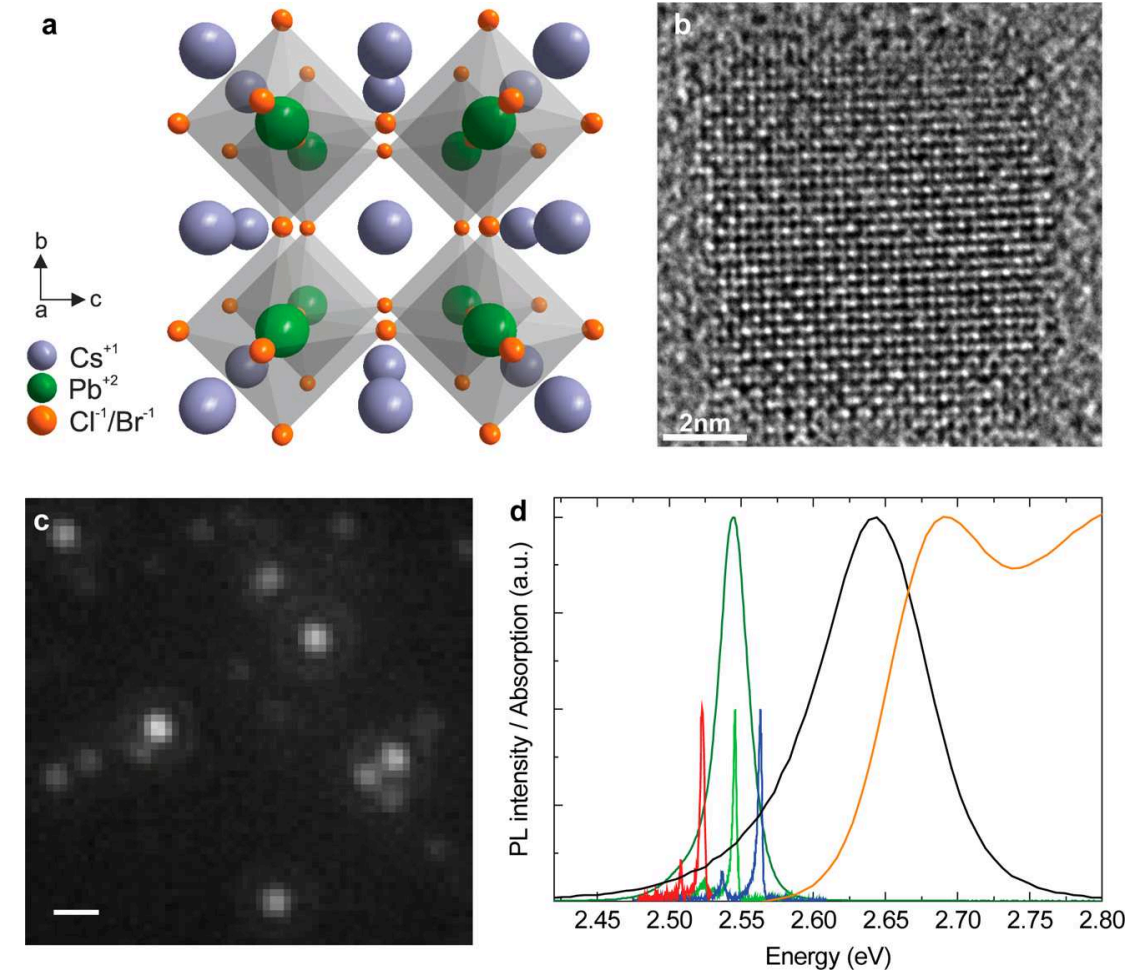

Figure 1. (a) Illustration of the crystal structure. (b) High-resolution transmission electron microscopy (HR-TEM) image of a single nanocrystal. (c) PL microscopy image from a low-density nanocrystal film obtained with (nonhomogeneous) wide-field excitation. The scale bar corresponds to $2 \mu \mathrm{m}$. (d) Absorption (orange) and PL (black) spectra from a high-density ensemble at room temperature, and the corresponding PL (dark green) at $T=6 \mathrm{~K}$. Representative spectra of three individual quantum dots in the low-density film (blue, green, red) at $T=6 \mathrm{~K}$ are superimposed.
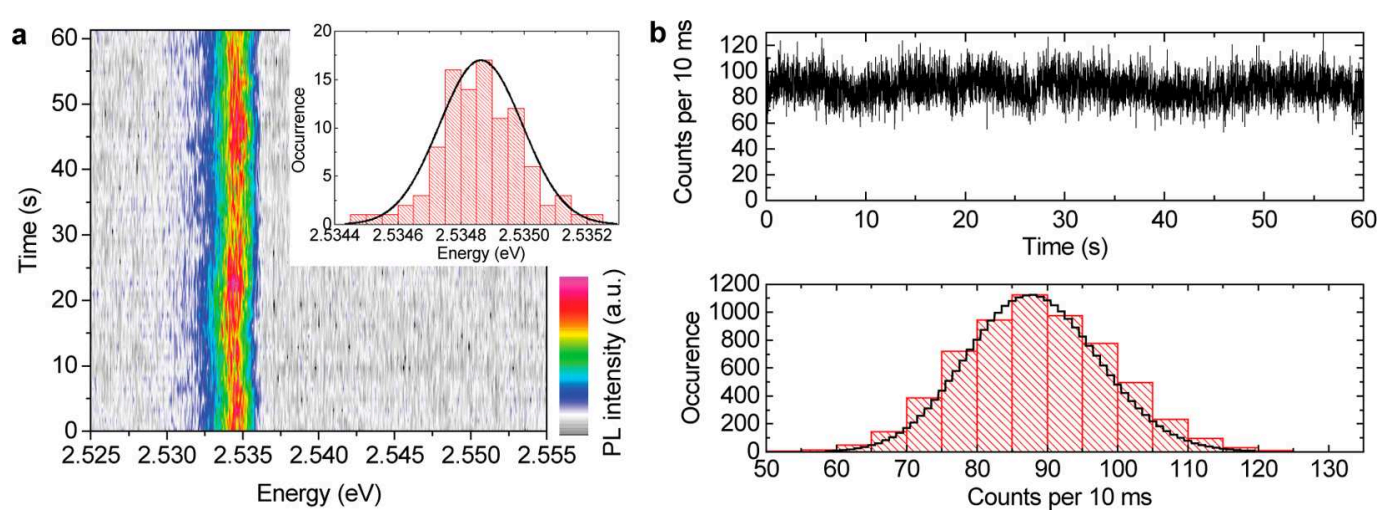

Figure 2. (a) PL spectral time series shows spectral diffusion. The inset is a histogram of the peak energies, which is described well by a Gaussian distribution (black line). (b) PL intensity as a function of time shows blinking-free emission. The histogram in the lower plot follows a Poissonian intensity distribution (black line).

commonly reported for hybrid organic-inorganic $\mathrm{MAPbX}_{3}$ thin-films, ${ }^{20}$ are clearly shared also by their $\mathrm{CsPbX}_{3} \mathrm{NCs}$ cousins showing high room-temperature photoluminescence quantum yields of up to $90 \%$ without any additional surface passivation. This opened up a wealth of opportunities for tunable light sources based on compositional and quantum-size tuning, ${ }^{19}$ including recently demonstrated single-photon emission at room temperature. ${ }^{21}$ These investigations clearly highlighted the immediate potential for real-world applications such as backlighting in television displays and light-emitting diodes. However, the broad emission line width at roomtemperature obscured many features of the underlying photophysics. Low temperature investigations could unveil a substantially different picture and provide deep insight into the fine structure, spectral diffusion and charged emitting states.

Here, we present a micro-photoluminescence study on single colloidal mixed-halide $\mathrm{CsPb}(\mathrm{Cl} / \mathrm{Br})_{3} \mathrm{NCs}$ of $c a .9 .5 \mathrm{~nm}$ diameter (Figure 1a-c, Methods and Supporting Information) at low temperature $(T=6 \mathrm{~K})$. In comparison to ensemble measurements of the same material, the spectral width of the photoluminescence (PL) is reduced from 25 to $1 \mathrm{meV}$ fullwidth at half-maximum (fwhm) due to the absence of inhomogeneous broadening (Figure 1d). The emission peak energy is red-shifted by $95 \mathrm{meV}$ compared to room temperature, due to the temperature-dependent deformation of the crystal lattice. Importantly, control experiments with Xray diffraction (XRD) confirmed retention of the cubic 

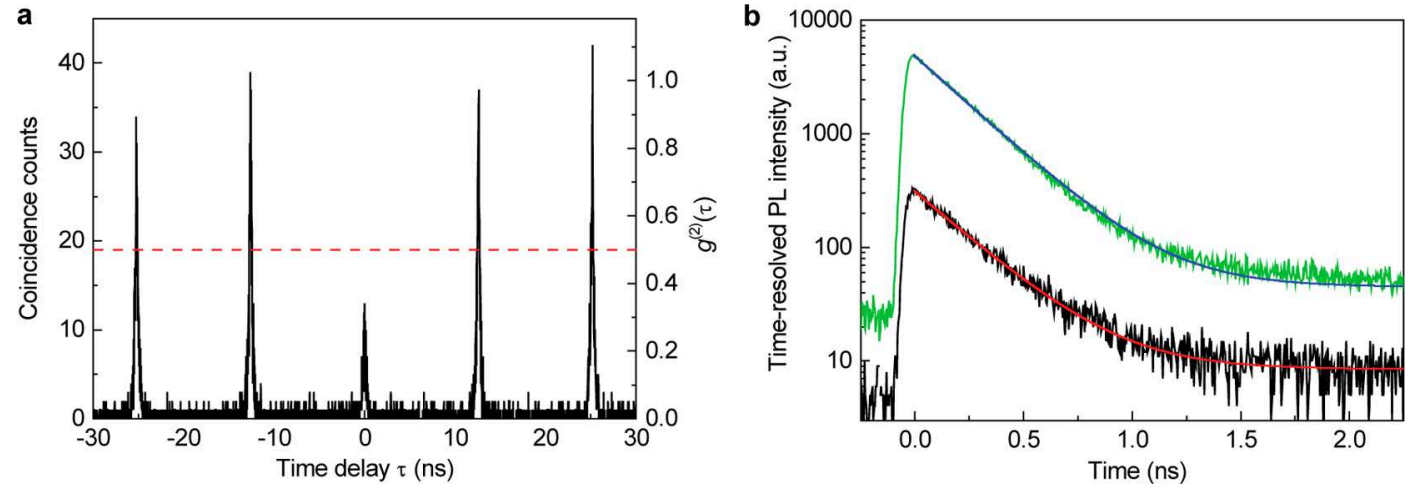

Figure 3. (a) The photon correlation obtained in a Hanbury-Brown-Twiss configuration shows peaks every $12.5 \mathrm{~ns}$ when the laser pulses excite the QD. Each peak is described by a single-exponential decay on both sides, representing the PL decay times. Single photon emission is manifested by the correlation peak at $\tau=0$ being suppressed to $g^{(2)}=0.3$ below the antibunching threshold at $g^{(2)}=0.5$. The plot shows raw data without background subtraction or deconvolution. (b) Time-resolved photon emission as a function of time after the excitation pulse (without background subtraction). The linear slope in the logarithmic plot for two different excitation powers $(0.8 \mu \mathrm{W}$, black and $7.5 \mu \mathrm{W}$, green) shows a power-independent single exponential decay over about 2 decades with a decay time of 250 ps (least-squares fits are red and blue, respectively).

perovskite crystal structure upon cooling (see Figure S2 in the Supporting Information), ruling out transition to tetragonal or orthorhombic phases as a factor influencing optical properties at low temperatures. The following results are representative of several samples from different synthesis batches, and are obtained from the measurements of tens of individual NCs.

\section{RESULTS}

In general, single quantum emitters are susceptible to their environment, and their emission can be altered by external electromagnetic fields. Within solid state systems, nearby trapped charges or polarization of the surrounding material lead to spectral diffusion of the emission energy. At low optical excitation power $P_{\mathrm{ex}}=1.8 \mu \mathrm{W}$, we observe spectral diffusion with a standard deviation of $0.13 \mathrm{meV}$, much below the PL fwhm of $1 \mathrm{meV}$ (Figure 2a). Statistics on the intensity of the emitted light with binning times of $10 \mathrm{~ms}$ show a nearly Poissonian intensity noise distribution (Figure $2 b$ ), reflecting a small non-Poissonian intensity flickering component and the absence of blinking events associated with dark states (see Figure S3 in the Supporting Information). While the magnitude of the spectral diffusion and also the presence of intensity flickering differ from $\mathrm{NC}$ to $\mathrm{NC}$, single perovskite $\mathrm{NC}$ in general show good photostability even without electronic passivation with a wider-gap shell material ${ }^{22-24}$ (see Figure S3 and Movie S1 in the Supporting Information).

The hallmark feature of quantum light sources is photon antibunching, i.e., that only single photons are emitted because the normalized second-order correlation function $g^{(2)}(\tau)$ drops to zero at $\tau=0$. We measure the photon correlations in a Hanbury-Brown-Twiss setup with pulsed excitation. In the experiment, we find $g^{(2)}(0)<0.5$ (Figure 3a) which unequivocally proves that the NCs are indeed zero-dimensional quantum dots (QDs) serving as deterministic single photon sources on demand. This also shows that we are investigating really single QDs since a cluster of $n$ QDs would yield ${ }^{25} g^{(2)}(0)$ $\geq 1-1 / n$. The magnitude of the antibunching depends on multiexciton generation within a single $\mathrm{QD}$ in addition to the background signal (autofluorescence of the substrate/matrix and components of the optical setup). We included more $g^{(2)}(\tau)$ traces in the Supporting Information (Figure S5) in order to rule-out other potential systematic effects. The difference between our values around $\sim 0.3$ at a temperature of $6 \mathrm{~K}$ and the 0.06 observed at room temperature in ref 21 could be due to a change of the efficiency to create or quench multiexciton states, ${ }^{26}$ e.g., via Auger recombination (see measurements for higher excitations power below). We observe that this value even raises at high excitation power (see Figure S6 in the Supporting Information), probably due to the presence of additional states being involved in the emission process.

We measured the PL lifetime as a function of $P_{\mathrm{ex}}$ (Figure $3 \mathrm{~b}$ ) and observe a single exponential decay over about 2 orders of magnitude which is characteristic of an effectively single excitonic transition. The $1 / e$ decay times slightly vary between different QDs within in the range of $180-250$ ps, which is significantly faster than for single molecules, ${ }^{11,27}$ epitaxially grown III- $\mathrm{V}^{13,28}$ or colloidal II- $\mathrm{VI}^{14}$ semiconductor QDs or nitrogen vacancy color centers in diamond. ${ }^{29}$ It is also $1-2$ orders of magnitude faster than at room temperature ${ }^{19,21}$ which could originate from an increased coherence volume of the exciton, as it is observed in many other systems such as semiconductor quantum wells ${ }^{30}$ or $\mathrm{CuCl}$ nanocrystals. ${ }^{31}$ Although a size-dependent study with NCs significantly smaller than the Bohr radius could help to pin down the origin for the fast decay, this is currently not feasible for single dot spectroscopy due to the tendency to crystal phase transitions and decomposition/aggregation upon isolation and purification with smaller NCs. The decay in the weak excitation regime below $10 \mu \mathrm{W}$ is almost independent of the excitation power, proving the absence of various quenching mechanisms such as Auger recombination or bimolecular annihilation of excitons. Subtracting the background from the substrate and the matrix (see Supporting Information Figure S4), we conclude that only a negligible fraction of luminescence $(\sim 10 \%$ of all detected photons) comes from very long-lived states (>10 ns). Because the room temperature quantum yield can reach $90 \%$, and the Auger-like power-dependent quenching is not observed, we attribute the short decay time to radiative recombination that reflects the high oscillator strength of the material.

Ideally, for a single quantum emitter the observed PL line width should be as narrow as the Fourier limit imposed by the emitter's decay time. Even for the lowest $P_{\text {ex }}$ the QD PL line widths appears to be more than an order of magnitude away 

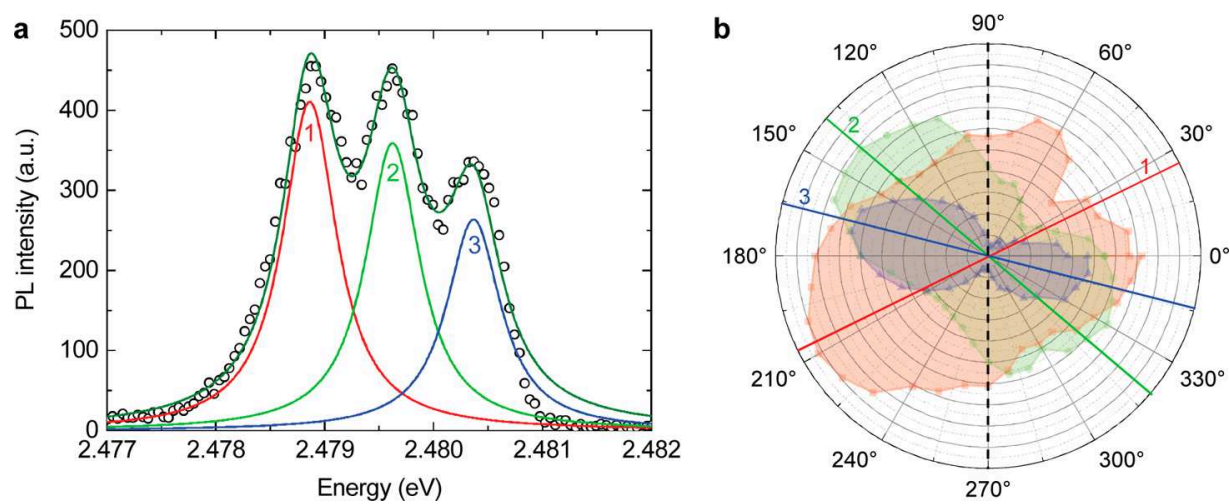

Figure 4. (a) At low excitation power $P_{\mathrm{ex}}$ and high spectral resolution with integration times of $10 \mathrm{~s}$ and a rotatable polarizer in the detection path, the PL spectrum reveals a complex fine structure. Three peaks (labeled 1, 2,3) can be resolved. (b) The polarization of each of the three peaks is measured by rotating the linear polarizer. The polar plot shows the intensities at the respective peak emission energy as a function of polarizer angle.
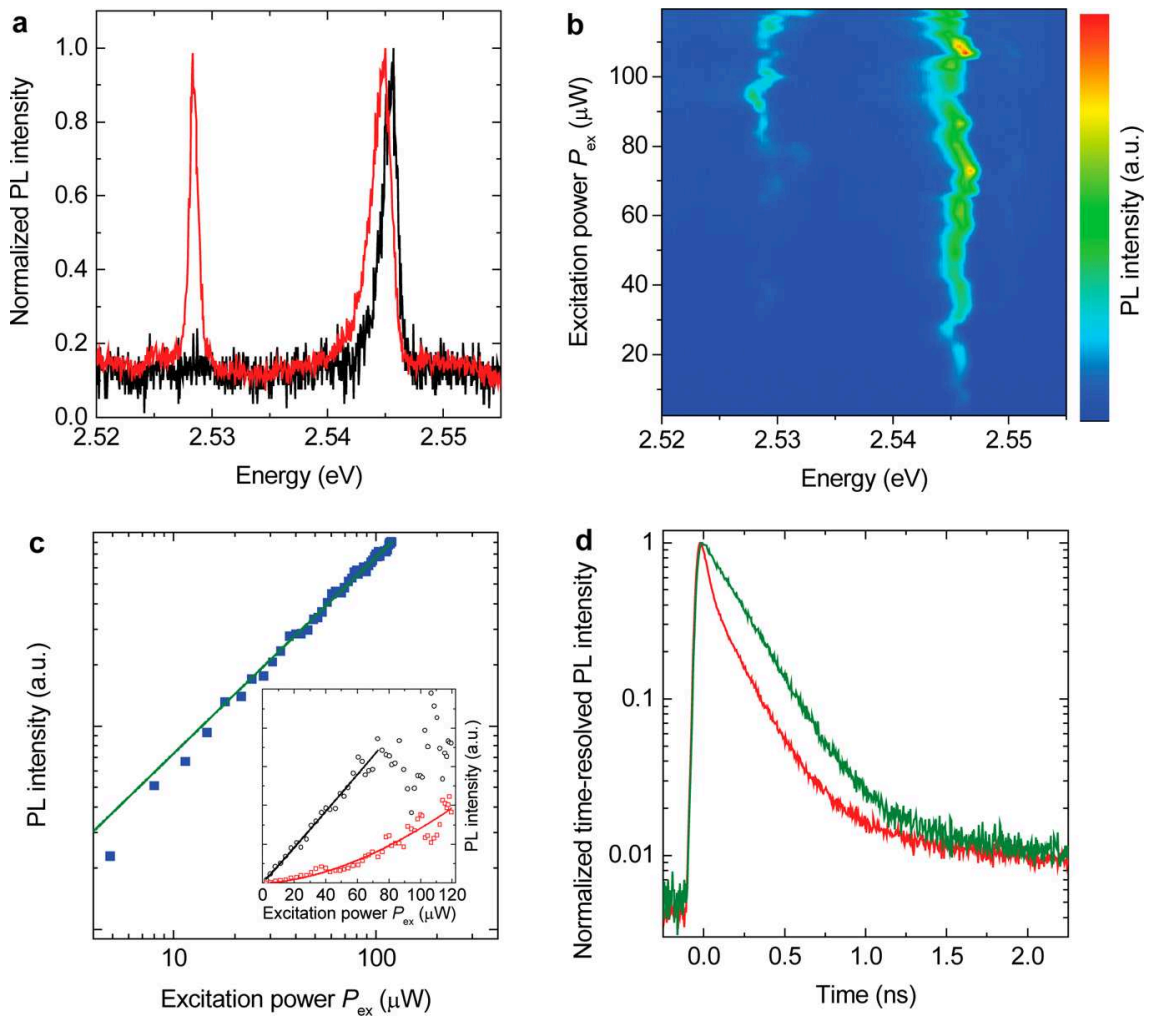

Figure 5. (a) Comparing low and high $P_{\mathrm{ex}}(11 \mu \mathrm{W}$, black, and $92 \mu \mathrm{W}$, red) an emission peak from charged excitons appears at lower energy. (b) PL as a function of $P_{\text {ex }}$ shows the gradual emergence of the charged exciton peak together with a strong increase in spectral diffusion and intermittency. (c) Spectrally integrated emission intensity as a function of excitation power shows linear behavior (power-law fit gives an exponent of $0.97 \pm 0.02)$ also in the high excitation regime. The inset shows power-law fits to the spectral ranges of the uncharged (black) and the charged exciton peaks (red), which yield exponents of $1 \pm 0.1$ (up to intermediate excitation power) and 1.5 \pm 0.1 , respectively. (d) Time-resolved PL measurement shows a nonexponential decay shortening for high excitation power (red, $51 \mu \mathrm{W}$ ) as compared to the weak excitation regime (green, 7.5 $\mu \mathrm{W}$ ). Both traces are raw data that is not corrected for the background.

from this limit. However, using high-resolution spectroscopy, we observe that the peak is asymmetric with a fwhm that varies from $\mathrm{QD}$ to $\mathrm{QD}$ between 0.6 and $2 \mathrm{meV}$, and for a fraction of the QDs a manifold of transitions is resolvable while photon antibunching is still observed. An exemplary case is shown in Figure $4 \mathrm{a}$, where three peaks with individual fwhm down to 0.6 $\mathrm{meV}$ are resolvable with an energy spacing of $0.75 \mathrm{meV}$ between them and amplitudes that decay toward higher energy. Polarization-resolved PL measurements reveal that they have different degrees and axes of polarization (Figure $4 \mathrm{~b}$ ). The peak widths, the splittings (if resolvable), and the relative polarization orientations vary from $\mathrm{QD}$ to $\mathrm{QD}$, suggesting that this fine structure is linked to anisotropies or defect complexes in the individual nanocrystals. ${ }^{32}$

Isolated single nanocrystals allow the investigation of photogeneration, recombination and trapping of charges to a degree that remains inaccessible within an ensemble of emitters where the inhomogeneous broadening washes out many features. While our experiments at low excitation power show a narrow single emission line with only little spectral diffusion 
and intermittency, this changes when the excitation power is increased (Figure 5a). At first, the excitonic emission peak still increases linearly with $P_{\mathrm{ex}}$, showing no density-induced quenching. Beyond a certain $P_{\mathrm{ex}}$ a second peak red-shifted by $16 \mathrm{meV}$ arises which intensity increases nonlinearly with $P_{\mathrm{ex}}{ }^{\alpha}$ with an exponent of $\alpha=1.5$ (inset Figure 5c). It also shows hysteretic behavior, i.e., that it can stay present sometimes for tens of seconds when the excitation power is lowered again. We attribute this additional emission peak to charged excitons, as the above behavior is a strong indication of photogenerated charges which can subsequently remain trapped in the QD. The amount of red-shift $(10-20 \mathrm{meV})$ and the exponent (1.3-1.7) vary between different QDs in the given ranges, suggesting that properties of the individual nanocrystals or surfaces like size, compositional variation $(\mathrm{Br} / \mathrm{Cl}$ ratio $)$ and defects play a role.

Furthermore, the spectral diffusion and intermittency of the uncharged and the charged exciton emission strongly increases with increasing $P_{\text {ex }}$ (Figure 5b and Figure S7b in the Supporting Information). At intermediate $P_{\mathrm{ex}}$ for some QDs the charged exciton line can become 2-3 times narrower than the exciton line, suggesting that the transition might have reduced electron-phonon coupling or vanishing fine structure splittings. At higher $P_{\mathrm{ex}}$, the charged exciton feature can split into multiple peaks that is most likely caused by multiple charges being generated (see Figure S7a in the Supporting Information). Most striking is the observation that the quantum yield (i.e., integrated PL intensity) of the majority of the QDs does not drop significantly in the high excitation regime (Figure 5c), while the decay at the same time is further accelerated and becomes nonexponential (Figure 5d). This suggests that, in contrast to room temperature, ${ }^{21}$ the faster decay is still mainly radiative and rather caused by the enhanced decay rates of charged or multiexcitons and not nonradiative Auger quenching.

\section{CONCLUSIONS}

In conclusion, our experiments demonstrate that colloidal NCs of metal-halide perovskites can serve as stable quantum emitters with some outstanding optical properties and versatile processability, allowing easy integration and offering a clear pathway for electrical driving. ${ }^{33}$ It is expected that the ultrafast radiative decay and single photon operation can enable strongcoupling cavity quantum electrodynamics experiments in high quality factor microcavities. ${ }^{34}$ In addition, high-resolution investigations on single NCs pave the way to a much deeper understanding of the photophysics of these exceptional materials, which can shed light on open questions such as the anomalous hysteresis. ${ }^{35}$ Furthermore, it can guide material development for optimized charge production and transport or core-shell/matrix heterostructures ${ }^{33}$ that are essential for a further leap in the efficiencies of solar cells ${ }^{1}$ and light sources. ${ }^{6}$

\section{METHODS}

Synthesis and Sample Preparation. $\mathrm{CsPb}(\mathrm{Cl} / \mathrm{Br})_{3} \mathrm{NCs}$ were synthesized by reacting Cs-oleate with a mixture of $\mathrm{PbCl}_{2}$ and $\mathrm{PbBr}_{2}$ in 1-octadecene at $200{ }^{\circ} \mathrm{C}$, as described previously ${ }^{19}$ (for details, see the Supporting Information). Isolation and purification procedures were optimized to yield nearly monodisperse $(10 \%)$ and aggregate-free colloids. The colloidal NCs were diluted to nanomolar concentrations within a solution of polystyrene in toluene. A thin film of $100 \mathrm{~nm}$ was then spin-cast on a high-purity, $3 \mu \mathrm{m}$ thick $\mathrm{SiO}_{2}$ layer which was obtained by thermally oxidizing a crystalline $\mathrm{Si}$ wafer.

Characterization. All measurements were performed in a homebuilt micro-PL setup with the sample mounted inside a liquid helium flow cryostat (Cryovac) in vacuum. For all photoluminescence measurements, a single-mode fiber-coupled continuous wave excitation laser at a wavelength of $\lambda=405 \mathrm{~nm}$ (Thorlabs) with linear polarization and short-pass filter (Semrock FF01-440/SP) was used. Excitation and detection was done through a long-working distance $100 \times$ microscope objective with numerical aperture of NA $=0.7$ (Mitutoyo Plan Apo NIR HR) which was mounted outside the cryostat. This resulted in a nearly Gaussian excitation spot with $1 / e^{2}$ radius of $1.4 \mu \mathrm{m}$. The emission from the sample was long-pass filtered (Semrock BLP01-442R), dispersed by an 1800 lines/mm grating in a $0.75 \mathrm{~m}$ monochromator (Acton Spectra Pro) and detected by a backilluminated cooled EMCCD camera (Princeton Instruments ProEM). For excitation in the photon correlation and PL lifetime measurements, a frequency-doubled Ti:sapphire laser at $\lambda=400 \mathrm{~nm}$ with 100 fs pulse duration and $80 \mathrm{MHz}$ repetition rate (Spectra-Physics Tsunami with Millennia pump laser) was coupled through a singlemode fiber to the setup. The detection was done after a tunable 80 meV-wide bandpass filter (Semrock TBP01-501/15) with a timecorrelated single photon counting system (PicoQuant PicoHarp 300 with PDM, MPD avalanche photo diodes with nominal time resolution of $30 \mathrm{ps})$.

\section{ASSOCIATED CONTENT}

\section{Supporting Information}

The Supporting Information is available free of charge on the ACS Publications website at DOI: 10.1021/acsnano.5b07328.

additional details and data on synthesis and characterization of $\mathrm{CsPb}(\mathrm{Cl} / \mathrm{Br})_{3} \mathrm{NCs}$, intensity flickering statistics, background subtraction for time-resolved PL measurements, additional photon antibunching data and optical characterization at higher excitation power (PDF) Movie S1 (AVI)

\section{AUTHOR INFORMATION}

\section{Corresponding Authors}

* gra@zurich.ibm.com (G.R.).

*mvkovalenko@ethz.ch (M.K.).

*tof@zurich.ibm.com (T.S.).

\section{Notes}

The authors declare no competing financial interest.

\section{ACKNOWLEDGMENTS}

We are grateful to B. J. Offrein and P. F. Seidler for insightful discussions, U. Drechsler and A. La Porta for help with the sample preparation and $\mathrm{M}$. Wörle for help with temperaturedependent XRD measurements. This work was partly supported by the European Union's Horizon-2020 and FP7 framework programmes through the Marie-Sklodowska Curie ITN network PHONSI (H2020-MSCA-ITN-642656) and ERC Starting Grant (NANOSOLID, GA No. 306733).

\section{REFERENCES}

(1) Grätzel, M. The Light and Shade of Perovskite Solar Cells. Nat. Mater. 2014, 13, 838-842.

(2) Stranks, S. D.; Snaith, H. J. Metal-Halide Perovskites for Photovoltaic and Light-Emitting Devices. Nat. Nanotechnol. 2015, 10, 391-402.

(3) Lee, M. M.; Teuscher, J.; Miyasaka, T.; Murakami, T. N.; Snaith, H. J. Efficient Hybrid Solar Cells Based on Meso-Superstructured Organometal Halide Perovskites. Science 2012, 338, 643-647.

(4) Nie, W.; Tsai, H.; Asadpour, R.; Blancon, J.-C.; Neukirch, A. J.; Gupta, G.; Crochet, J. J.; Chhowalla, M.; Tretiak, S.; Alam, M. A.; Wang, H.-L.; Mohite, A. D. High-Efficiency Solution-Processed Perovskite Solar Cells with Millimeter-Scale Grains. Science 2015, 347, 522-525. 
(5) Jeon, N. J.; Noh, J. H.; Yang, W. S.; Kim, Y. C.; Ryu, S.; Seo, J.; Seok, S. I. Compositional Engineering of Perovskite Materials for High-Performance Solar Cells. Nature 2015, 517, 476-480.

(6) Tan, Z.-K.; Moghaddam, R. S.; Lai, M. L.; Docampo, P.; Higler, R.; Deschler, F.; Price, M.; Sadhanala, A.; Pazos, L. M.; Credgington, D.; Hanusch, F.; Bein, T.; Snaith, H. J.; Friend, R. H. Bright LightEmitting Diodes Based on Organometal Halide Perovskite. Nat. Nanotechnol. 2014, 9, 687-692.

(7) Xing, G.; Mathews, N.; Lim, S. S.; Yantara, N.; Liu, X.; Sabba, D.; Grätzel, M.; Mhaisalkar, S.; Sum, T. C. Low-Temperature SolutionProcessed Wavelength-Tunable Perovskites for Lasing. Nat. Mater. 2014, 13, 476-480.

(8) Zhang, Q.; Ha, S. T.; Liu, X.; Sum, T. C.; Xiong, Q. RoomTemperature Near-Infrared High-Q Perovskite Whispering-Gallery Planar Nanolasers. Nano Lett. 2014, 14, 5995-6001.

(9) Deschler, F.; Price, M.; Pathak, S.; Klintberg, L. E.; Jarausch, D.D.; Higler, R.; Hüttner, S.; Leijtens, T.; Stranks, S. D.; Snaith, H. J.; Atatüre, M.; Phillips, R. T.; Friend, R. H. High Photoluminescence Efficiency and Optically Pumped Lasing in Solution-Processed Mixed Halide Perovskite Semiconductors. J. Phys. Chem. Lett. 2014, 5, 14211426.

(10) Wen, X.; Ho-Baillie, A.; Huang, S.; Sheng, R.; Chen, S.; Ko, H.C.; Green, M. A. Mobile Charge-Induced Fluorescence Intermittency in Methylammonium Lead Bromide Perovskite. Nano Lett. 2015, 15, 4644-4649.

(11) Basché, T.; Moerner, W. E.; Orrit, M.; Talon, H. Photon Antibunching in the Fluorescence of a Single Dye Molecule Trapped in a Solid. Phys. Rev. Lett. 1992, 69, 1516-1519.

(12) Empedocles, S. A.; Norris, D. J.; Bawendi, M. G. Photoluminescence Spectroscopy of Single CdSe Nanocrystallite Quantum Dots. Phys. Rev. Lett. 1996, 77, 3873-3876.

(13) Michler, P.; Kiraz, A.; Becher, C.; Schoenfeld, W. V.; Petroff, P. M.; Zhang, L.; Hu, E.; Imamoglu, A. A Quantum Dot Single-Photon Turnstile Device. Science 2000, 290, 2282-2285.

(14) Michler, P.; Imamoglu, A.; Mason, M. D.; Carson, P. J.; Strouse, G. F.; Buratto, S. K. Quantum Correlation Among Photons from a Single Quantum Dot at Room Temperature. Nature 2000, 406, 968970.

(15) Kurtsiefer, C.; Mayer, S.; Zarda, P.; Weinfurter, H. Stable SolidState Source of Single Photons. Phys. Rev. Lett. 2000, 85, 290-293.

(16) Brouri, R.; Beveratos, A.; Poizat, J.-P.; Grangier, P. Photon Antibunching in the Fluorescence of Individual Color Centers in Diamond. Opt. Lett. 2000, 25, 1294-1296.

(17) Betzig, E.; Patterson, G. H.; Sougrat, R.; Lindwasser, O. W.; Olenych, S.; Bonifacino, J. S.; Davidson, M. W.; Lippincott-Schwartz, J.; Hess, H. F. Imaging Intracellular Fluorescent Proteins at Nanometer Resolution. Science 2006, 313, 1642-1645.

(18) Eisaman, M. D.; Fan, J.; Migdall, A.; Polyakov, S. V. SinglePhoton Sources and Detectors. Rev. Sci. Instrum. 2011, 82, 071101.

(19) Protesescu, L.; Yakunin, S.; Bodnarchuk, M. I.; Krieg, F.; Caputo, R.; Hendon, C. H.; Yang, R. X.; Walsh, A.; Kovalenko, M. V. Nanocrystals of Cesium Lead Halide Perovskites (CsPbX3, X $=\mathrm{Cl}, \mathrm{Br}$, and I): Novel Optoelectronic Materials Showing Bright Emission with Wide Color Gamut. Nano Lett. 2015, 15, 3692-3696.

(20) Oga, H.; Saeki, A.; Ogomi, Y.; Hayase, S.; Seki, S. Improved Understanding of the Electronic and Energetic Landscapes of Perovskite Solar Cells: High Local Charge Carrier Mobility, Reduced Recombination, and Extremely Shallow Traps. J. Am. Chem. Soc. 2014, 136, 13818-13825.

(21) Park, Y.-S.; Guo, S.; Makarov, N. S.; Klimov, V. I. Room Temperature Single-Photon Emission from Individual Perovskite Quantum Dots. ACS Nano 2015, 9, 10386-10393.

(22) Frantsuzov, P.; Kuno, M.; Jankó, B.; Marcus, R. A. Universal Emission Intermittency in Quantum Dots, Nanorods and Nanowires. Nat. Phys. 2008, 4, 519-522.

(23) Mahler, B.; Spinicelli, P.; Buil, S.; Quelin, X.; Hermier, J.-P.; Dubertret, B. Towards non-blinking colloidal quantum dots. Nat. Mater. 2008, 7, 659-664.
(24) Galland, C.; Ghosh, Y.; Steinbrück, A.; Sykora, M.; Hollingsworth, J. A.; Klimov, V. I.; Htoon, H. Two Types of Luminescence Blinking Revealed by Spectroelectrochemistry of Single Quantum Dots. Nature 2011, 479, 203-208.

(25) Brouri, R.; Beveratos, A.; Poizat, J.-P.; Grangier, P. Photon Antibunching in the Fluorescence of Individual Color Centers in Diamond. Opt. Lett. 2000, 25, 1294-1296.

(26) Nair, G.; Zhao, J.; Bawendi, M. G. Biexciton Quantum Yield of Single Semiconductor Nanocrystals from Photon Statistics. Nano Lett. 2011, 11, 1136-1140.

(27) Lounis, B.; Moerner, W. E. Single Photons on Demand from a Single Molecule at Room Temperature. Nature 2000, 407, 491-493.

(28) Shields, A. Semiconductor Quantum Light Sources. Nat. Photonics 2007, 1, 215-223.

(29) Doherty, M. W.; Manson, N. B.; Delaney, P.; Jelezko, F.; Wrachtrup, J.; Hollenberg, L. C. L. The Nitrogen-Vacancy Colour Centre in Diamond. Phys. Rep. 2013, 528, 1.

(30) Feldmann, J.; Peter, G.; Göbel, E. O.; Dawson, P.; Moore, K.; Foxon, C.; Elliott, R. J. Linewidth Dependence of Radiative Exciton Lifetimes in Quantum Wells. Phys. Rev. Lett. 1987, 59, 2337-2340.

(31) Itoh, T.; Furumiya, M.; Ikehara, T.; Gourdon, C. SizeDependent Radiative Decay Time of Confined Excitons in $\mathrm{CuCl}$ Microcrystals. Solid State Commun. 1990, 73, 271-274.

(32) Buin, A.; Comin, R.; Xu, J.; Ip, A. H.; Sargent, E. H. HalideDependent Electronic Structure of Organolead Perovskite Materials. Chem. Mater. 2015, 27, 4405-4412.

(33) Ning, Z.; Gong, X.; Comin, R.; Walters, G.; Fan, F.; Voznyy, O.; Yassitepe, E.; Buin, A.; Hoogland, S.; Sargent, E. H. Quantum-Dot-inPerovskite Solids. Nature 2015, 523, 324-328.

(34) Kimble, H. J. The Quantum Internet. Nature 2008, 453, 10231030.

(35) Snaith, H. L.; Abate, A.; Ball, J. M.; Eperon, G. E.; Leijtens, T.; Noel, N. K.; Stranks, S. D.; Wang, J. T.-W.; Wojciechowski, K.; Zhang, W. Anomalous Hysteresis in Perovskite Solar Cells. J. Phys. Chem. Lett. 2014, 5, 1511-1515. 